\title{
Multipliers for Jacobi expansions in the Hardy spaces
}

\section{Zhong Xue ${ }^{1}$, Yehao Shi ${ }^{2}$ and Zhongkai Lij ${ }^{3^{*}}$}

"Correspondence: lizk@shnu.edu.cn ${ }^{3}$ Department of Mathematics, Shanghai Normal University, Shanghai, China

Full list of author information is available at the end of the article

\begin{abstract}
The purpose of the paper is to study the coefficient multipliers of the Hardy spaces $H^{P}$ associated with Jacobi expansions of exponential type. The main results are about the boundedness from $H^{p}$ to $\ell^{q}$ of the multiplier operators in terms of Jacobi expansions of exponential type for (i) $p=1,2 \leq q<\infty$; (ii) $\gamma(\alpha, \beta)^{-1}<p<1 \leq q<\infty$, under appropriate conditions, where $\gamma(\alpha, \beta) \in(1, \infty]$ is a number depending on the parameters of the Jacobi system.
\end{abstract}

MSC: Primary 42A45; secondary 46E15; 42C10

Keywords: Hardy space; Multipliers; Jacobi expansion

\section{Introduction and main results}

\subsection{Jacobi expansions of exponential type}

Assume that $\alpha, \beta>-1$. Let $R_{n}^{(\alpha, \beta)}(x)$ be the Jacobi polynomial on $[-1,1]$ of degree $n$ normalized so that $R_{n}^{(\alpha, \beta)}(1)=1$. It follows that the system $\left\{R_{n}^{(\alpha, \beta)}(\cos t)\right\}_{n=0}^{\infty}$ is orthogonal over $[0, \pi]$ with respect to the weight $\sin ^{2 \alpha+1}(t / 2) \cos ^{2 \beta+1}(t / 2)$. In particular,

$$
R_{n}^{(-1 / 2,-1 / 2)}(\cos t)=\cos n t, \quad R_{n}^{(1 / 2,1 / 2)}(\cos t)=\frac{\sin (n+1) t}{(n+1) \sin t} .
$$

In analogy to the relation of $\cos n t$ and $\sin n t$, the system $\left\{R_{n-1}^{(\alpha+1, \beta+1)}(\cos t) \sin t\right\}_{n=1}^{\infty}$ is introduced in [8], which is a conjugate one of $\left\{R_{n}^{(\alpha, \beta)}(\cos t)\right\}_{n=0}^{\infty}$ based on a pair of generalized Cauchy-Riemann equations. This allows us to define an exponential type system $\left\{E_{n}^{(\alpha, \beta)}(t)\right\}_{n=-\infty}^{\infty}$ as in [9], by $E_{0}^{(\alpha, \beta)}=1 / \sqrt{2}$, and for $n \geq 1$,

$$
\begin{aligned}
E_{n}^{(\alpha, \beta)}(t) & =\frac{1}{2}\left[R_{n}^{(\alpha, \beta)}(\cos t)+i \frac{\rho_{n}}{2 \alpha+2} R_{n-1}^{(\alpha+1, \beta+1)}(\cos t) \sin t\right], \\
E_{-n}^{(\alpha, \beta)}(t) & =\overline{E_{n}^{(\alpha, \beta)}(t)},
\end{aligned}
$$

with $\rho_{n}=\sqrt{n(n+\alpha+\beta+1)}$, which is orthogonal over $[-\pi, \pi]$ with respect to the weight $w(t)=\phi_{\alpha, \beta}(t)^{2}$, where

$$
\phi_{\alpha, \beta}(t)=|\sin (t / 2)|^{\alpha+1 / 2}|\cos (t / 2)|^{\beta+1 / 2} \text {. }
$$

(c) The Author(s) 2020. This article is licensed under a Creative Commons Attribution 4.0 International License, which permits use, sharing, adaptation, distribution and reproduction in any medium or format, as long as you give appropriate credit to the original author(s) and the source, provide a link to the Creative Commons licence, and indicate if changes were made. The images or other third party material in this article are included in the article's Creative Commons licence, unless indicated otherwise in a credit line to the material. If material is not included in the article's Creative Commons licence and your intended use is not permitted by statutory regulation or exceeds the permitted use, you will need to obtain permission directly from the copyright holder. To view a copy of this licence, visit http://creativecommons.org/licenses/by/4.0/. 
It is interesting that each $E_{n}^{(\alpha, \beta)}$ is an eigenfunction of the first order differential-difference operator $T$ defined by

$$
T f(t)=\frac{d}{d t} f(t)+\eta_{\alpha, \beta}(t)[f(t)-f(-t)], \quad \eta_{\alpha, \beta}(t)=\frac{(\alpha+\beta+1) \cos t+\alpha-\beta}{2 \sin t},
$$

that is,

$$
T E_{ \pm n}^{(\alpha, \beta)}(t)= \pm i \rho_{n} E_{ \pm n}^{(\alpha, \beta)}(t) \quad \text { for } n \geq 0 .
$$

This resembles the functions $e^{i n t}$ satisfying $\frac{d}{d t} e^{ \pm i n t}= \pm i n e^{ \pm i n t}$. The system $\left\{E_{n}^{(\alpha, \beta)}(t)\right\}_{n=-\infty}^{\infty}$ induces an orthonormal system $\left\{\mathcal{E}_{n}^{(\alpha, \beta)}(t)\right\}_{n=-\infty}^{\infty}$ over $[-\pi, \pi]$ with respect to the Lebesgue measure, that is,

$$
\int_{-\pi}^{\pi} \mathcal{E}_{m}^{(\alpha, \beta)}(t) \overline{\mathcal{E}_{n}^{(\alpha, \beta)}(t)} d t=\delta_{m, n}, \quad m, n=0, \pm 1, \pm 2, \ldots
$$

where, for $n=0, \pm 1, \pm 2, \ldots$,

$$
\mathcal{E}_{n}^{(\alpha, \beta)}(t)=\sqrt{\omega_{n}^{(\alpha, \beta)}} E_{n}^{(\alpha, \beta)}(t) \phi_{\alpha, \beta}(t),
$$

and $1 / \omega_{n}^{(\alpha, \beta)}=\int_{-\pi}^{\pi}\left|E_{n}^{(\alpha, \beta)}(t)\right|^{2} \phi_{\alpha, \beta}(t)^{2} d t$. From [9, Lemma 4] and [8, (2.2)] it follows that, for $n=0,1,2, \ldots$,

$$
\omega_{n}^{(\alpha, \beta)}=\frac{(2 n+\alpha+\beta+1) \Gamma(n+\alpha+\beta+1) \Gamma(n+\alpha+1)}{\Gamma(\alpha+1) \Gamma(\alpha+1) \Gamma(n+\beta+1) \Gamma(n+1)},
$$

and $\omega_{-n}^{(\alpha, \beta)}=\omega_{n}^{(\alpha, \beta)}$. It is easy to see that

$$
\omega_{n}^{(\alpha, \beta)}=2 \Gamma(\alpha+1)^{-2} n^{2 \alpha+1}\left(1+O\left(n^{-1}\right)\right) \quad \text { for } n \geq 1 .
$$

Sometimes we write $E_{n}(t)=E_{n}^{(\alpha, \beta)}(t)$ and $\mathcal{E}_{n}(t)=\mathcal{E}_{n}^{(\alpha, \beta)}(t)$ for simplicity.

In what follows we assume that $\alpha, \beta \geq-1 / 2$, for which the functions $\mathcal{E}_{n}^{(\alpha, \beta)}(t)$ are continuous on $[-\pi, \pi]$. For $f \in L(-\pi, \pi)$, its Jacobi expansion of exponential type is defined by

$$
f(t) \sim \sum_{n=-\infty}^{\infty} c_{n}(f) \mathcal{E}_{n}^{(\alpha, \beta)}(t), \quad c_{n}(f)=\int_{-\pi}^{\pi} f(t) \overline{\mathcal{E}_{n}^{(\alpha, \beta)}(t)} d t
$$

where $c_{n}(f)$ are called the Fourier-Jacobi coefficients of $f$.

\subsection{The main results}

The purpose of the paper is to study the coefficient multipliers of the real Hardy spaces $H^{p}(-\pi, \pi)$ associated with Jacobi expansions of exponential type. We recall that a function $F$ analytic in the unit disk $\mathbb{D}$ is said to be in the Hardy space $H^{p}(\mathbb{D}), 0<p<\infty$, if $\|F\|_{H^{p}}:=$ $\sup _{0 \leq r<1} M_{p}(F ; r)<\infty$, where

$$
M_{p}(F ; r)=\left\{\frac{1}{2 \pi} \int_{-\pi}^{\pi}\left|F\left(r e^{i \theta}\right)\right|^{p} d \theta\right\}^{1 / p} .
$$


The real Hardy space $H^{p}(-\pi, \pi)$ consists of boundary values of real parts of functions $F$ in $H^{p}(\mathbb{D})$, with real $F(0)$.

Since $H^{1}(-\pi, \pi) \subset L(-\pi, \pi)$, the Fourier-Jacobi coefficients of $f \in H^{1}(-\pi, \pi)$ may be defined as in (3); but if $f \in H^{p}(-\pi, \pi)$ for $0<p<1$, we need a substitute definition of its Fourier-Jacobi coefficients $c_{n}(f)$, which is based on the duality relation of the Hardy space $H^{p}(-\pi, \pi)$ and the Lipschitz space $\Lambda_{p^{-1}-1}(-\pi, \pi)$. For $m \geq 1$ and $m-1<\delta \leq m, \Lambda_{\delta}(-\pi, \pi)$ is the set of $(m-1)$-times differentiable and $2 \pi$-period functions $f$ satisfying

$$
\|f\|_{\Lambda_{\delta}}:=\sup _{x, h}\left|f^{(m-1)}(x+h)-f^{(m-1)}(x)\right| /|h|^{\delta+1-m}<\infty
$$

for $\delta \neq m$, and

$$
\|f\|_{\Lambda_{\delta}}:=\sup _{x, h}\left|f^{(m-1)}(x+h)-2 f^{(m-1)}(x)+f^{(m-1)}(x-h)\right| /|h|<\infty
$$

for $\delta=m$. Here we use a unified notation $\Lambda_{\delta}(-\pi, \pi)$ for all $\delta>0$, without use of Zygmund's notation $\Lambda_{\delta}^{*}(-\pi, \pi)$ for $\delta=m$.

Lemma 1.1 ([1, Theorem 7.5]) To each bounded linear functional $\mathcal{L}$ on $H^{p}(\mathbb{D}), 0<p<1$, there is a function $g \in \Lambda_{p^{-1}-1}(-\pi, \pi)$ such that, for all $F(z)=\sum_{n=0}^{\infty} c_{n} z^{n} \in H^{p}(\mathbb{D})$,

$$
\mathcal{L}(F)=\lim _{r \rightarrow 1-} \int_{-\pi}^{\pi} F\left(r e^{i t}\right) g(t) d t
$$

Conversely, for any $g \in \Lambda_{p^{-1}-1}(-\pi, \pi)$, the above limit exists for all $F \in H^{p}(\mathbb{D})$ and defines a bounded linear functional satisfying

$$
|\mathcal{L}(F)| \leq c\|g\|_{\Lambda_{p^{-1}-1}}\|F\|_{H^{p}}
$$

where $c$ is a constant independent of $g$ and $F$.

A convenient notation is $\mathcal{L}_{g}=\mathcal{L}$ once $\mathcal{L}$ and $g$ satisfy relation (4).

The linear functional $\mathcal{L}$ on the real Hardy space $H^{p}(-\pi, \pi), 0<p<1$, is identified with the associated one on $H^{p}(\mathbb{D})$, that means $\mathcal{L}(f)=\mathcal{L}(F)$, where $f(t)=$ the real part of $F\left(e^{i t}\right)$ for $F \in H^{p}(\mathbb{D})$ with real $F(0)$.

For $0<p<1$, the Fourier-Jacobi coefficients $c_{n}(f)$ of $f \in H^{p}(-\pi, \pi)$ are defined by

$$
c_{n}(f)=\mathcal{L} \overline{\mathcal{E}_{n}^{(\alpha, \beta)}}(f) \quad \text { for } n=0, \pm 1, \pm 2, \ldots
$$

It is easy to see that this definition is identical with the previous definition in (3) for "good" functions. However, it is not always meaningful in general for all $H^{p}(-\pi, \pi), 0<p<1$, since the functions $\mathcal{E}_{n}^{(\alpha, \beta)}(t)$ are not sufficiently smooth for most of $\alpha, \beta$. Indeed we have the following.

Proposition 1.2 Let $\alpha, \beta \geq-1 / 2$. The functions $\mathcal{E}_{n}(t)=\mathcal{E}_{n}^{(\alpha, \beta)}(t)$ are in $\Lambda_{p^{-1}-1}(-\pi, \pi)$ for $\gamma(\alpha, \beta)^{-1} \leq p<1$, where

$$
\begin{aligned}
\gamma(\alpha, \beta) & =+\infty \quad \text { if both } \alpha+1 / 2 \text { and } \beta+1 / 2 \text { are nonnegative even integers } \\
& =\min \{\alpha, \beta\}+3 / 2 \quad \text { if neither } \alpha+1 / 2 \text { nor } \beta+1 / 2 \text { is an even integer }
\end{aligned}
$$




$$
\begin{gathered}
=\alpha+3 / 2 \text { or } \beta+3 / 2 \quad \text { if } \alpha+1 / 2 \text { or } \beta+1 / 2 \text { is not an even integer } \\
\text { and the other one is an even integer. }
\end{gathered}
$$

Consequently, for $f \in H^{p}(-\pi, \pi), \gamma(\alpha, \beta)^{-1} \leq p<1$, its Fourier-Jacobi coefficients $c_{n}(f)=$ $\mathcal{L} \frac{\mathcal{E}_{n}^{(\alpha, \beta)}}{(f)}$ are well defined.

It is obvious that $\gamma(\alpha, \beta)>1$ for all $\alpha, \beta \geq-1 / 2$.

The main results in the present paper are about the boundedness of the multiplier operators associated with Jacobi expansions of exponential type from the Hardy spaces $H^{p}(-\pi, \pi)$ to the sequence spaces $\ell^{q}$ for some ranges of $p$ and $q$. The details are stated in the following two theorems.

Theorem 1.3 Let $\alpha, \beta \geq-1 / 2$ and $2 \leq q<\infty$. If a bilateral sequence $\left\{\lambda_{n}\right\}_{n=-\infty}^{\infty}$ satisfies the condition

$$
\sum_{N \leq|n| \leq 2 N}\left|\lambda_{n}\right|^{q}=O(1) \quad \text { for } N \geq 1
$$

then, for all $f \in H^{1}(-\pi, \pi)$ having the Jacobi expansion (3) of exponential type,

$$
\sum_{n=-\infty}^{\infty}\left|\lambda_{n} c_{n}(f)\right|^{q} \leq c\|f\|_{H^{1}}^{q}
$$

Theorem 1.4 Let $\alpha, \beta \geq-1 / 2$ and

$$
\gamma(\alpha, \beta)^{-1}<p<1 \leq q<\infty
$$

If a bilateral sequence $\left\{\lambda_{n}\right\}_{n=-\infty}^{\infty}$ satisfies the condition

$$
\sum_{N \leq|n| \leq 2 N}\left|\lambda_{n}\right|^{q}=O\left(N^{q(1-1 / p)}\right) \quad \text { for } N \geq 1
$$

then, for all $f \in H^{p}(-\pi, \pi)$, the Fourier-Jacobi coefficients $c_{n}(f)=\mathcal{L}_{\overline{\mathcal{E}_{n}^{(\alpha, \beta)}}}(f)$ satisfy

$$
\sum_{n=-\infty}^{\infty}\left|\lambda_{n} c_{n}(f)\right|^{q} \leq c\|f\|_{H^{p}}^{q} .
$$

As a consequence of Theorems 1.3 and 1.4, a Paley-type inequality associated with Jacobi expansions (3) can be obtained. We first note that if $\left\{n_{k}\right\}$ is a Hadamard sequence satisfying $n_{k+1} / n_{k} \geq \rho>1(k=1,2, \ldots)$, then for any $N=1,2, \ldots$, the number of elements in $\left\{n_{k}\right\}$ locating in $[N, 2 N]$ has a bound independent of $N$. Indeed, if $n_{k-1}<N \leq n_{k}$, then for $j \geq$ $k$ satisfying $n_{j} \leq 2 N$, we have $1 \leq 2 N n_{j}^{-1} \leq 2 N n_{k}^{-1} \rho^{k-j} \leq 2 \rho^{k-j}$, and hence the bound to be determined is $2 /\left(1-\rho^{-1}\right)$. Now if $\lambda_{n}=n_{k}^{1-1 / p}$ for $|n|=n_{k}$, and 0 otherwise, then the sequence $\left\{\lambda_{n}\right\}_{n=-\infty}^{\infty}$ satisfies (5) or (7) for $p=1$ or $0<p<1$ respectively, and hence applying Theorems 1.3 and 1.4 to $q=2$, we have the following corollary. 
Corollary 1.5 Let $\alpha, \beta \geq-1 / 2$ and $\gamma(\alpha, \beta)^{-1}<p \leq 1$. If $\left\{n_{k}\right\}$ is a Hadamard sequence satisfying $n_{k+1} / n_{k} \geq \rho>1(k=1,2, \ldots)$, then for $f \in H^{p}(-\pi, \pi)$, the Fourier-Jacobi coefficients $c_{n}(f)$ satisfy

$$
\sum_{k=1}^{\infty} n_{k}^{2(1-1 / p)}\left(\left|c_{n_{k}}(f)\right|^{2}+\left|c_{-n_{k}}(f)\right|^{2}\right) \leq c\|f\|_{H^{p}}^{2}
$$

The Paley-type inequality for usual Jacobi expansions is a special case of (9) with $p=1$ and for even functions, which has been proved in [7] by the duality of $H^{1}$ and BMO.

Throughout the paper, $c$ or $c^{\prime}$ denotes constants independent of variables, functions, $n$, $k$, etc., which may be different in different occurrences.

\subsection{Backgrounds and remarks}

1. The research on multipliers for power series and Fourier series has a long history and rich contents. One of the criteria of multipliers in the Hardy spaces, proved in Hardy and Littlewood [4,5], is that, for $1 \leq p \leq 2 \leq q$ and $p^{-1}-q^{-1}=1-\delta^{-1}$, the sequence $\left\{\lambda_{n}\right\}$ is a multiplier of $H^{p}(\mathbb{D})$ into $H^{q}(\mathbb{D})$ if the function $h_{\lambda}(z)=\sum_{k=0}^{\infty} \lambda_{k} z^{k}$ satisfies

$$
M_{\delta}\left(h_{\lambda}^{\prime} ; r\right) \leq c(1-r)^{-1}
$$

It was pointed out in [13] (see [12] too) that (10) with $\delta=q$ is also necessary for the sequence $\left\{\lambda_{n}\right\}$ being a multiplier of $H^{1}(\mathbb{D})$ into $H^{q}(\mathbb{D})$ when $1 \leq q \leq \infty$, and hence, for $q \geq 2$, it provides a characterization of multipliers from $H^{1}(\mathbb{D})$ into $H^{q}(\mathbb{D})$. In particular, by Parseval's theorem, (10) for $\delta=2$ is equivalent to

$$
\sum_{n=N}^{2 N}\left|\lambda_{n}\right|^{2}=O(1) \quad \text { for } N \geq 1 .
$$

That means the sequence $\left\{\lambda_{n}\right\}$ is a multiplier of $H^{1}(\mathbb{D})$ into $H^{2}(\mathbb{D})$ if and only if $(11)$ is satisfied.

Turning to the case for $1 \leq p \leq q<2$, the situation is completely different from (10). A sequence $\left\{\lambda_{n}\right\}$ is constructed in [12] (see Theorem 3.1 there), which satisfies (10) but is not a multiplier of $H^{p}(\mathbb{D})$ into $H^{q}(\mathbb{D})$. However, it was shown in [2] (and first stated in $[4,5])$ that a modification of (10) allows to extend the theorem of Hardy and Littlewood to smaller $p$. Indeed, if $0<p<1 \leq q \leq \infty$ and $(v+1)^{-1} \leq p<v^{-1}$ with $v=1,2, \ldots$, then the sequence $\left\{\lambda_{n}\right\}$ is a multiplier of $H^{p}(\mathbb{D})$ into $H^{q}(\mathbb{D})$ if and only if the function $h_{\lambda}(z)=$ $\sum_{n=0}^{\infty} \lambda_{n} z^{n}$ satisfies $M_{q}\left(h_{\lambda}^{(\nu+1)} ; r\right) \leq c(1-r)^{\frac{1}{p}-\nu-2}$.

A different type of multipliers is the coefficient multipliers of the Hardy spaces $H^{p}(\mathbb{D})$ into the sequence spaces $\ell^{q}$. For $0<q<\infty, \ell^{q}=\left\{\left\{a_{k}\right\}:\left\|\left\{a_{k}\right\}\right\|_{q}=\left(\sum_{k=0}^{\infty}\left|a_{k}\right|^{q}\right)^{1 / q}<\infty\right\}$; and $\ell^{\infty}$ is the set of bounded sequences. We use the same notations for a bilateral sequence. That a sequence $\left\{\lambda_{n}\right\}$ is such a multiplier means that $\left\{\lambda_{n} c_{n}\right\} \in \ell^{q}$ whenever $\sum_{n=0}^{\infty} c_{n} z^{n} \in$ $H^{p}(\mathbb{D})$.

A basic criterion following from that on multipliers of $H^{1}(\mathbb{D})$ into $H^{2}(\mathbb{D})\left(\right.$ alias $\left.\ell^{2}\right)$ mentioned above is stated as follows: the sequence $\left\{\lambda_{n}\right\}$ is a multiplier of $H^{1}(\mathbb{D})$ into $\ell^{q}$ for $2 \leq q<\infty$ if and only if $\sum_{n=N}^{2 N}\left|\lambda_{n}\right|^{q}=O(1)$ for $N \geq 1$. For details, see [2, pp. 72-73]. A general extension of this criterion to the case $0<p<1$ is given in [2]. It is proved in [2] that (see 
Theorem 2 there), for $0<p<1$ and $p \leq q<\infty$, the sequence $\left\{\lambda_{n}\right\}$ is a multiplier of $H^{p}(\mathbb{D})$ into $\ell^{q}$ if and only if $\sum_{n=N}^{2 N}\left|\lambda_{n}\right|^{q}=O\left(N^{q(1-1 / p)}\right)$ for $N \geq 1$. We note that this condition is equivalent to what is given in [2] by the following proposition.

Proposition 1.6 ([10, Proposition 1.8]) Let $a, b$ be real and $a>0$. Then, for a nonnegative sequence $\left\{\mu_{n}\right\}$, the following conditions are equivalent:

(i) $\sum_{n=1}^{N} n^{b} \mu_{n}=O\left(N^{a}\right)$ for $N \geq 1$;

(ii) $\sum_{n=N}^{2 N} \mu_{n}=O\left(N^{a-b}\right)$ for $N \geq 1$;

(iii) $\sum_{n=2^{k}}^{2^{k+1}} \mu_{n}=O\left(2^{k(a-b)}\right)$ for $k \geq 0$;

(iv) For some $\delta>0, \sum_{n=N}^{\infty} n^{b-a-\delta} \mu_{n}=O\left(N^{-\delta}\right)$ for $N \geq 1$.

Since an element in the real Hardy spaces $H^{p}(-\pi, \pi)$ has an expansion associated with $\left\{e^{i n \theta}\right\}_{n=-\infty}^{\infty}$, or equivalently with $\{1, \cos n \theta, \sin n \theta, n=1,2, \ldots\}$, the coefficient multiplier criteria given above can be restated as follows.

\section{Theorem 1.7}

(i) If a bilateral sequence $\left\{\lambda_{n}\right\}_{n=-\infty}^{\infty}$ satisfies condition (5), then it is a multiplier of $H^{1}(-\pi, \pi)$ into $\ell^{q}$ for $2 \leq q<\infty$, which means $\left\{\lambda_{n} c_{n}\right\} \in \ell^{q}$ whenever $\sum_{n=-\infty}^{\infty} c_{n} e^{i n t} \in H^{1}(-\pi, \pi)$.

(ii) Suppose $0<p<1$. If a bilateral sequence $\left\{\lambda_{n}\right\}_{n=-\infty}^{\infty}$ satisfies condition (7), then it is a multiplier of $H^{p}(-\pi, \pi)$ into $\ell^{q}$ for $p \leq q<\infty$, which means $\left\{\lambda_{n} c_{n}\right\} \in \ell^{q}$ whenever $\sum_{n=-\infty}^{\infty} c_{n} e^{i n t} \in H^{p}(-\pi, \pi)$.

2. If $f \in L(-\pi, \pi)$ is even or $f \in L(0, \pi)$, (3) is identical with the following usual Jacobi expansion:

$$
f(t) \sim \sum_{n=0}^{\infty} a_{n}(f) p_{n}^{(\alpha, \beta)}(t), \quad a_{n}(f)=\int_{0}^{\pi} f(t) p_{n}^{(\alpha, \beta)}(t) d t
$$

where

$$
p_{n}^{(\alpha, \beta)}(t)=\sqrt{\omega_{n}^{(\alpha, \beta)}} R_{n}^{(\alpha, \beta)}(\cos t) \phi_{\alpha, \beta}(t), \quad n=0,1,2, \ldots
$$

which form an orthonormal system over $[0, \pi]$ with respect to the Lebesgue measure.

3. In our previous work [10], the problem on coefficient multipliers of the Hardy spaces $H^{p}(\mathbb{R})$ associated with Hermite expansions was studied. A sufficient condition given in [10] for a sequence $\left\{\lambda_{n}\right\}_{n=0}^{\infty}$ to be a multiplier of $H^{p}(\mathbb{R})$ into the sequence space $\ell^{q}$ associated with Hermite expansions for (i) $p=1,2 \leq q<\infty$ and (ii) $0<p<1 \leq q<\infty$ is

$$
\sum_{k=n}^{2 n}\left|\lambda_{k}\right|^{q}=O\left(n^{\frac{q}{2}\left(\frac{7}{6}-\frac{1}{p}\right)}\right) .
$$

In comparison to (5) and (7), condition (12) might seem peculiar. For a Hadamard sequence $\left\{n_{k}\right\}$, a Paley-type inequality following (12) is of the form (see [10, Corollary 3.3])

$$
\sum_{k=1}^{\infty} n_{k}^{\frac{7}{6}-\frac{1}{p}}\left|a_{n_{k}}(f)\right|^{2} \leq c\|f\|_{H^{p}(\mathbb{R})}^{2}, \quad f \in H^{p}(\mathbb{R}),
$$


where $a_{n}(f), n \geq 0$, are the coefficients of the Hermite expansion of $f$. It is noted that the Hardy inequality associated with Hermite expansions was proved in [11], that is,

$$
\sum_{n=1}^{\infty} n^{-\frac{3}{4}}\left|a_{n}(f)\right| \leq c \mid f \|_{H^{1}(\mathbb{R})}, \quad f \in H^{1}(\mathbb{R}) .
$$

The sharpness of (14) was verified by Kanjin in [6], who showed that there exists $f_{0} \in L^{1}(\mathbb{R})$ such that

$$
\sum_{n=1}^{\infty} n^{-\frac{3}{4}}\left|a_{n}\left(f_{0}\right)\right|=\infty
$$

4. The original proofs of the classical multiplier theorems depend on the complex variable structure of analytic functions, which is not workable for the Jacobi expansions of exponential type. We shall apply, in Sect. 3, the duality of $H^{1}$ and BMO in proving Theorem 1.3, and the duality of $H^{p}(-\pi, \pi)$ and $\Lambda_{p^{-1}-1}(-\pi, \pi)$ in the proof of Theorem 1.4. Applications of these principles base upon some evaluations of the exponential type Jacobi functions $\mathcal{E}_{n}^{(\alpha, \beta)}(t)$ which are given in Sect. 2.

\section{Several lemmas}

Lemma 2.1 Let $\alpha, \beta>-1$ and let $0<\epsilon_{0}<\pi$ be fixed. Then, for $0 \leq|t| \leq \pi-\epsilon_{0}$ and for $n=1,2, \ldots$,

$$
\mathcal{E}_{n}^{(\alpha, \beta)}(t)=\frac{\sqrt{\omega_{n}^{(\alpha, \beta)}}}{2}\left(\frac{|t|}{2}\right)^{\alpha+1 / 2} e_{\alpha+1 / 2}(i N t)+O\left[\frac{1}{n}\left(\frac{n|t|}{1+n|t|}\right)^{\alpha+3 / 2}\right]
$$

with $N=n+(\alpha+\beta+1) / 2$, where $e_{\lambda}$ is the one-dimensional Dunkl kernel

$$
e_{\lambda}(z)=j_{\lambda-1 / 2}(i z)+\frac{z}{2 \lambda+1} j_{\lambda+1 / 2}(i z), \quad z \in \mathbb{C},
$$

and $j_{\alpha}(z)$ is the normalized Bessel function $j_{\alpha}(z)=2^{\alpha} \Gamma(\alpha+1) z^{-\alpha} J_{\alpha}(z)$.

Proof The proof is essentially based upon the well-known formula [14, (8.21.17)] of "Hilb's type", which is rewritten as

$$
\phi_{\alpha, \beta}(t) R_{n}^{(\alpha, \beta)}(\cos t)=\left(\frac{t}{2}\right)^{\alpha+1 / 2} j_{\alpha}(N t)+O\left[\left(\frac{t}{1+n t}\right)^{\alpha+3 / 2}\right]
$$

for $0 \leq t \leq \pi-\epsilon_{0}$. Applying to $R_{n-1}^{(\alpha+1, \beta+1)}(\cos t)$ and noting that $\rho_{n}=N+O(1)$, then equality (15) follows from (1)-(2) and these estimates. If $-\pi+\epsilon_{0} \leq t \leq 0$, we use the relation $\mathcal{E}_{n}^{(\alpha, \beta)}(t)=\overline{\mathcal{E}_{n}^{(\alpha, \beta)}(-t)}$ to obtain (15) again.

Corollary 2.2 Let $\alpha, \beta>-1$ and let $0<\epsilon_{0}<\pi$ be fixed. Then, for $n^{-1} \leq|t| \leq \pi-\epsilon_{0}$ and for $n=1,2, \ldots$,

$$
\begin{aligned}
\mathcal{E}_{n}^{(\alpha, \beta)}(t)= & \frac{1}{\sqrt{2 \pi}}\left[\left(1+i \frac{(2 \alpha+1)^{2}}{8 N t}\right) e^{i(\operatorname{sgn} t)\left(N|t|-\frac{\pi}{2} \alpha-\frac{\pi}{4}\right)}\right. \\
& \left.+i \frac{2 \alpha+1}{4 N t} e^{-i(\operatorname{sgn} t)\left(N|t|-\frac{\pi}{2} \alpha-\frac{\pi}{4}\right)}\right]+O\left(\frac{1}{n^{2} t^{2}}+\frac{1}{n}\right) .
\end{aligned}
$$


Proof We rewrite $[3,7-13(3)]$ as

$$
j_{\alpha}(x)=\frac{c_{\alpha+1 / 2}}{|x|^{\alpha+1 / 2}}\left[\cos \left(|x|-\frac{\pi}{2} \alpha-\frac{\pi}{4}\right)+\frac{1-4 \alpha^{2}}{8|x|} \sin \left(|x|-\frac{\pi}{2} \alpha-\frac{\pi}{4}\right)+O\left(\frac{1}{x^{2}}\right)\right]
$$

for $x \rightarrow \infty$, where $c_{\lambda}=2^{\lambda} \Gamma(\lambda+1 / 2) / \sqrt{\pi}$. Applying to $j_{\lambda-1 / 2}$ and $j_{\lambda+1 / 2}$, then from (16) it follows that

$$
e_{\lambda}(i x)=\frac{c_{\lambda}}{|x|^{\lambda}}\left[\left(1+i \frac{\lambda^{2}}{2 x}\right) e^{i(\operatorname{sgn} x)\left(|x|-\frac{\pi}{2} \lambda\right)}+i \frac{\lambda}{2 x} e^{-i(\operatorname{sgn} x)\left(|x|-\frac{\pi}{2} \lambda\right)}+O\left(\frac{1}{x^{2}}\right)\right] .
$$

Putting $\lambda=\alpha+1 / 2, x=N t$, and then substituting into (15) proves (17) on account of (2).

Lemma 2.3 Let $\alpha, \beta>-1$ and let $0<\epsilon_{0}<\pi$ be fixed. Then, for $k=0,1, \ldots$ and $0 \leq|t| \leq$ $\pi-\epsilon_{0}$,

$$
\left|\frac{d^{k}}{d t^{k}} E_{n}^{(\alpha, \beta)}(t)\right| \leq c n^{k}(1+n|t|)^{-\alpha-1 / 2}
$$

where $c$ is a constant independent of $n, t$.

Proof The key step is the following equality:

$$
\frac{d^{k}}{d t^{k}}\left[R_{n}^{(\alpha, \beta)}(\cos t)\right]=\sum_{j=0}^{k} U_{j}(n) R_{n-j}^{(\alpha+j, \beta+j)}(\cos t) \psi_{j}(t),
$$

where $U_{j}(n)$ is a polynomial in $n$ of degree $2 j$ and $U_{j}(n) \asymp n^{2 j}$ as $n \rightarrow \infty, \psi_{j}(t)$ is a homogeneous polynomial in $\cos t$ and $\sin t$ of degree $j$, and when $[(k+1) / 2] \leq j \leq k, \psi_{j}(t)=$ $(\sin t)^{2 j-k} \times$ is a homogeneous polynomial in $\cos t$ and $\sin t$ of degree $k-j$.

The equality in (19) is a consequence of the formula (see [8, (2.9)])

$$
\frac{d}{d x} R_{n}^{(\alpha, \beta)}(x)=\frac{\rho_{n}^{2}}{2 \alpha+2} R_{n-1}^{(\alpha+1, \beta+1)}(x)
$$

after induction. And hence, by means of the estimate $\left|R_{n}^{(\alpha, \beta)}(\cos t)\right| \leq c(1+|n t|)^{-\alpha-1 / 2}$ (see $[14,(7.32 .5)]),\left|\frac{d^{k}}{d t^{k}}\left[R_{n}^{(\alpha, \beta)}(\cos t)\right]\right|$ is dominated by a multiple of

$$
\sum_{j=[(k+1) / 2]}^{k} \frac{n^{2 j}|t|^{2 j-k}}{(1+|n t|)^{j+\alpha+1 / 2}}+\sum_{j=0}^{[(k-1) / 2]} \frac{n^{2 j}}{(1+|n t|)^{j+\alpha+1 / 2}}
$$

and consequently by $c n^{k}(1+n|t|)^{-\alpha-1 / 2}$, since $n^{2 j}|t|^{2 j-k} \leq n^{k}(1+|n t|)^{2 j-k}$ in the first summation and $n^{2 j} \leq n^{k}$ in the second one. Furthermore, we also have

$$
\begin{aligned}
\frac{\rho_{n}}{2 \alpha+2}\left|\left[R_{n-1}^{(\alpha+1, \beta+1)}(\cos t) \sin t\right]_{t}^{(k)}\right| & =\frac{1}{\rho_{n}}\left|\left[R_{n}^{(\alpha, \beta)}(\cos t)\right]_{t}^{(k+1)}\right| \\
& \leq c n^{k}(1+n|t|)^{-\alpha-1 / 2}
\end{aligned}
$$

and then by (1) the desired estimate (18) follows immediately. 
Lemma 2.4 Let $\alpha, \beta \geq-1 / 2$ and let $0<\epsilon_{0}<\pi$ be fixed. Then there is a constant $c$ independent of $n, t$ such that

$$
\left|\frac{d^{k}}{d t^{k}} \mathcal{E}_{n}^{(\alpha, \beta)}(t)\right| \leq c n^{k}\left(\frac{|n t|}{1+|n t|}\right)^{\alpha-k+1 / 2}
$$

for (i) $0 \leq k<\alpha+1 / 2,0 \leq|t| \leq \pi-\epsilon_{0}$, and (ii) $k \geq \alpha+1 / 2,0<|t| \leq \pi-\epsilon_{0}$. Furthermore, (iii) if $\alpha+1 / 2$ is an even integer, then $\left|\frac{d^{k}}{d t^{k}} \mathcal{E}_{n}^{(\alpha, \beta)}(t)\right| \leq c n^{k}$ for $k \geq \alpha+1 / 2$ and $0 \leq|t| \leq \pi-\epsilon_{0}$.

Proof By Leibnitz's rule,

$$
\frac{d^{k}}{d t^{k}} \mathcal{E}_{n}^{(\alpha, \beta)}(t)=\sqrt{\omega_{n}^{(\alpha, \beta)}} \sum_{j=0}^{k}\left(\begin{array}{l}
k \\
j
\end{array}\right) E_{n}^{(k-j)}(t) \phi_{\alpha, \beta}^{(j)}(t)
$$

It is obvious that $\phi_{\alpha, \beta}^{(j)}(t)=|\sin (t / 2)|^{\alpha+1 / 2-j} h(t)$, where $h(t)$ is a continuous function for $0 \leq$ $|t| \leq \pi-\epsilon_{0}$. We have $\left|\phi_{\alpha, \beta}^{(j)}(t)\right| \leq c|t|^{\alpha+1 / 2-j}$ for $t \neq 0$ and also for $t=0$ when $j<\alpha+1 / 2$, and $\left|\phi_{\alpha, \beta}^{(j)}(t)\right| \leq c$ for $j \geq \alpha+1 / 2$ when $\alpha+1 / 2$ is an even integer. Now by Lemma 2.3 we obtain

$$
\left|\frac{d^{k}}{d t^{k}} \mathcal{E}_{n}^{(\alpha, \beta)}(t)\right| \leq c n^{\alpha+1 / 2} \sum_{j=0}^{k} \frac{n^{k-j}|t|^{\alpha-j+1 / 2}}{(1+n|t|)^{\alpha+1 / 2}} \leq c n^{k}\left(\frac{|n t|}{1+|n t|}\right)^{\alpha-k+1 / 2}
$$

for $0<|t| \leq \pi-\epsilon_{0}$ and also for $t=0$ when $k<\alpha+1 / 2$. If $\alpha+1 / 2$ is an even integer and $k \geq \alpha+1 / 2$, again by Lemma 2.3 the summation with $j<\alpha+1 / 2$ is, as above, bounded by $c n^{k}$, and the remainder part is dominated by

$$
c n^{\alpha+1 / 2} \sum_{j=\alpha+1 / 2}^{k} n^{k-j}(1+n|t|)^{-\alpha-1 / 2} \leq c n^{k} .
$$

Combining all the evaluations finishes the proof of the lemma.

Corollary 2.5 Let $\alpha, \beta \geq-1 / 2$. Then, for $0 \leq k<\gamma(\alpha, \beta)-1$,

$$
\left|\frac{d^{k}}{d t^{k}} \mathcal{E}_{n}^{(\alpha, \beta)}(t)\right| \leq c n^{k}
$$

The corollary is a consequence of Lemma 2.4 and the relation

$$
\mathcal{E}_{n}^{(\alpha, \beta)}(t)=(-1)^{n} \mathcal{E}_{n}^{(\beta, \alpha)}(\pi-t) .
$$

Lemma 2.6 Let $\alpha, \beta \geq-1 / 2$. Then if $1 \leq m<\gamma(\alpha, \beta)-1$,

$$
\left|\mathcal{E}_{n}^{(m-1)}(s)-\mathcal{E}_{n}^{(m-1)}(t)\right| \leq c n^{m}|s-t| ;
$$

if $\gamma(\alpha, \beta)$ is finite and $\gamma(\alpha, \beta)-1 \leq m<\gamma(\alpha, \beta)$, then

$$
\left|\mathcal{E}_{n}^{(m-1)}(s)-\mathcal{E}_{n}^{(m-1)}(t)\right| \leq c n^{\gamma(\alpha, \beta)-1}|s-t|^{\gamma(\alpha, \beta)-m} .
$$


Proof For $1 \leq m<\gamma(\alpha, \beta)-1,(22)$ is a consequence of the mean-value theorem and Corollary 2.5 .

In what follows we assume that $\gamma(\alpha, \beta)$ is finite and $\gamma(\alpha, \beta)-1 \leq m<\gamma(\alpha, \beta)$. If $\mid s-$ $t \mid \geq(2 n)^{-1}$, the estimate is obvious since $\left|\mathcal{E}_{n}^{(m-1)}(t)\right| \leq c n^{m-1}$. Now we consider the case when $|s-t| \leq(2 n)^{-1}(\leq \pi / 6)$. Furthermore, we assume that $\gamma(\alpha, \beta)=\alpha+3 / 2$ and restrict ourselves to the case for $-\pi / 4 \leq t \leq 3 \pi / 4$, which implies $-5 \pi / 12 \leq s \leq 11 \pi / 12$. By (20), $\left|\mathcal{E}_{n}^{(m-1)}(s)-\mathcal{E}_{n}^{(m-1)}(t)\right|$ is dominated by a multiple of $n^{\alpha+1 / 2}$ times

$$
\left|E_{n}(s) \phi_{\alpha, \beta}^{(m-1)}(s)-E_{n}(t) \phi_{\alpha, \beta}^{(m-1)}(t)\right|+\sum_{j=0}^{m-2}\left|\left(E_{n}^{(m-1-j)}(t) \phi_{\alpha, \beta}^{(j)}(t)\right)_{t=\xi_{1}}^{\prime}\right||s-t|
$$

with some $\xi_{1}$ lying between $s$ and $t$. The second term above is bounded by, applying Lemma 2.3,

$$
\begin{aligned}
& \sum_{j=0}^{m-2}\left|E_{n}^{(m-j)}\left(\xi_{1}\right) \phi_{\alpha, \beta}^{(j)}\left(\xi_{1}\right)+E_{n}^{(m-1-j)}\left(\xi_{1}\right) \phi_{\alpha, \beta}^{(j+1)}\left(\xi_{1}\right)\right||s-t| \\
& \quad \leq c \sum_{j=0}^{m-2}\left[\frac{n^{m-j}\left|\xi_{1}\right|^{\alpha-j+1 / 2}}{\left(1+\left|n \xi_{1}\right|\right)^{\alpha+1 / 2}}+\frac{n^{m-j-1}\left|\xi_{1}\right|^{\alpha-j-1 / 2}}{\left(1+\left|n \xi_{1}\right|\right)^{\alpha+1 / 2}}\right]|s-t| \\
& \quad \leq c n^{m-\alpha-1 / 2}|s-t|
\end{aligned}
$$

since $\left|n \xi_{1}\right|^{\alpha-j-1 / 2} \leq\left(1+\left|n \xi_{1}\right|\right)^{\alpha-j-1 / 2}$ for $j \leq m-2$. In the meantime, it is easy to see that the first term in (24) is bounded by

$$
\left|E_{n}^{\prime}\left(\xi_{2}\right)\right|\left|\phi_{\alpha, \beta}^{(m-1)}(t)\right||s-t|+\left|E_{n}(s)\right|\left|\phi_{\alpha, \beta}^{(m-1)}(s)-\phi_{\alpha, \beta}^{(m-1)}(t)\right|
$$

for some $\xi_{2}$ lying between $s$ and $t$. Since

$$
\phi_{\alpha, \beta}^{(m-1)} \in \operatorname{Lip}(\alpha-m+3 / 2), \quad 0<\alpha-m+3 / 2 \leq 1,
$$

and $\left(1+\left|n \xi_{2}\right|\right) \asymp(1+|n t|)$ for $s, t$ under consideration, again applying Lemma 2.3 we obtain an upper bound of the first term in (24) as a multiple of

$$
\frac{n|t|^{\alpha-m+3 / 2}}{\left(1+\left|n \xi_{2}\right|\right)^{\alpha+1 / 2}}|s-t|+|s-t|^{\alpha-m+3 / 2} \leq c n^{m-\alpha-1 / 2}|s-t|+|s-t|^{\alpha-m+3 / 2} .
$$

Substituting the two estimates into (24) yields

$$
\left|\mathcal{E}_{n}^{(m-1)}(s)-\mathcal{E}_{n}^{(m-1)}(t)\right| \leq c n^{\alpha+1 / 2}|s-t|^{\alpha-m+3 / 2}
$$

for $|s-t| \leq(2 n)^{-1}$ and $\alpha+1 / 2 \leq m<\alpha+3 / 2$.

If $3 \pi / 4 \leq t \leq 7 \pi / 4$ or $\gamma(\alpha, \beta)=\beta+3 / 2$, the associated estimate in (23) is a consequence of the proved case and formula (21).

Lemma 2.7 Let $\alpha, \beta \geq-1 / 2$. There exists a constant c such that, for all interval $I,|I| \leq \pi / 4$,

$$
\left|\int_{I} \mathcal{E}_{k}(t) \mathcal{E}_{j}(t) d t\right| \leq c\left(\frac{|j|}{|k|}|I|+\frac{1}{|k|}\right) .
$$


Proof If $|k| / 2 \leq|j| \leq|k|$, then by Corollary $2.5,\left|\int_{I} \mathcal{E}_{k}(t) \mathcal{E}_{j}(t) d t\right| \leq c|I|$. In what follows, we assume that $|j| \leq|k| / 2$. Since $|I| \leq \pi / 4$, we may suppose $I \subseteq[-3 \pi / 4,3 \pi / 4]$, and the case when $I \subseteq[\pi / 4,7 \pi / 4]$ has a similar result by appealing to (21). If $I$ contains 0 as an interior point, then we divide it by 0 into two parts, and hence we may assume $I \subseteq[0,3 \pi / 4]$. We also assume that $k, j>0$ without loss of generality. At first we have

$$
\left|\int_{I \cap\left\{t: t \leq k^{-1}\right\}} \mathcal{E}_{k}(t) \mathcal{E}_{j}(t) d t\right| \leq c k^{-1} \quad \text { by Corollary } 2.5
$$

It remains to show that, for $j \leq k / 2$,

$$
\left|\int_{I \cap\left\{t: t \geq k^{-1}\right\}} \mathcal{E}_{k}(t) \mathcal{E}_{j}(t) d t\right| \leq c\left(\frac{j}{k}|I|+\frac{1}{k}\right) .
$$

We apply (17) to the $\mathcal{E}_{k}(t)$ in (25). The contribution of the $O$-term, in conjunction with Corollary 2.5 , to the integral is dominated by

$$
c \int_{k^{-1}}^{3 \pi / 4}\left[(k t)^{-2}+k^{-1}\right] d t \leq c^{\prime} k^{-1}
$$

We need to evaluate the critical part of the integral in (25) according to (17), that is,

$$
A_{j, k}:=\int_{I \cap\left\{t: t \geq k^{-1}\right\}} e^{i K t} \mathcal{E}_{j}(t) d t
$$

where $K=k+(\alpha+\beta+1) / 2$. But the evaluation of the part of the integral associated with the terms in (17) with additional factor $(K t)^{-1}$ is a little easier.

Taking integration by parts yields

$$
A_{j, k}=\frac{i}{K} \int_{I \cap\left\{t: t \geq k^{-1}\right\}} e^{i K t} \mathcal{E}_{j}^{\prime}(t) d t+O\left(k^{-1}\right) .
$$

By Lemma $2.4,\left|\mathcal{E}_{j}^{\prime}(t)\right| \leq c j$ for $\alpha=-1 / 2$ and $\left|\mathcal{E}_{j}^{\prime}(t)\right| \leq c j(j t /(1+j t))^{\alpha-1 / 2}$ for $\alpha>-1 / 2$. It follows from (26) that, for $\alpha=-1 / 2,\left|A_{j, k}\right| \leq c(j|I|+1) / k$, and for $\alpha>1 / 2$,

$$
\left|A_{j, k}\right| \leq c \frac{j}{K} \int_{I}\left(\frac{j t}{1+j t}\right)^{\alpha-1 / 2} d t+O\left(k^{-1}\right)
$$

It is obvious that the integration over $I$ is dominated by a multiple of

$$
\int_{0}^{j^{-1}}(j t)^{\alpha-1 / 2} d t+\int_{I} d t \leq c^{\prime}\left(j^{-1}+|I|\right)
$$

and immediately one has an upper bound for $\left|A_{j, k}\right|$ as in (25), as desired.

\section{Proofs of the main results}

\subsection{Proof of Theorem 1.3}

We first note that the conclusion for $2<q<\infty$ follows from that for $q=2$. Indeed, if we put $v_{n}=\left|\lambda_{n}\right|^{q / 2}$, then (5) implies $\sum_{N \leq|n| \leq 2 N}\left|v_{n}\right|^{2}=O(1)$, and since $\left|c_{k}(f)\right| \leq c\|f\|_{H^{1}}$ by 
Corollary 2.5 with $k=0$, we have

$$
\sum_{k=-\infty}^{\infty}\left|\lambda_{k} c_{k}(f)\right|^{q} \leq c^{\prime}\|f\|_{H^{1}}^{q-2} \sum_{k=-\infty}^{\infty}\left|v_{k} c_{k}(f)\right|^{2} \leq c\|f\|_{H^{1}}^{q}
$$

Now we turn to the proof of the theorem for $q=2$. We fix a sequence $\left\{b_{n}\right\}_{n=-\infty}^{\infty} \in \ell^{2}$ and for $n=1,2, \ldots$, put

$$
g_{n}(t)=\sum_{k=-n}^{n} \lambda_{k} b_{k} \overline{\mathcal{E}_{k}(t)}
$$

In terms of the duality of $H^{1}(-\pi, \pi)$ and $B M O$, one has $\left|\int_{-\pi}^{\pi} f(t) g_{n}(t) d t\right| \leq c\left\|g_{n}\right\|_{B M O}\|f\|_{H^{1}}$, or equivalently,

$$
\left|\sum_{k=-n}^{n} \lambda_{k} b_{k} c_{k}(f)\right| \leq c\left\|g_{n}\right\|_{B M O}\|f\|_{H^{1}}
$$

where $\|g\|_{B M O}=\sup _{I}(1 /|I|) \int_{I}\left|g(t)-g_{I}\right| d t$ for taking $I$ to be all interval of the line and $g_{I}=(1 /|I|) \int_{I} g(t) d t$ with $|I|$ being the length of $I$. We shall show that

$$
\left\|g_{n}\right\|_{B M O} \leq c^{\prime}\left(\sum_{k=-n}^{n}\left|b_{k}\right|^{2}\right)^{1 / 2}
$$

for a constant $c^{\prime}$ independent of $n$ and $\left\{b_{k}\right\}_{k=-\infty}^{\infty} \in \ell^{2}$. Once (29) is true, then from (28) it follows that $\left(\sum_{k=-n}^{n}\left|\lambda_{k} c_{k}(f)\right|^{2}\right)^{1 / 2} \leq c\|f\|_{H^{1}}$, which proves the theorem by letting $n \rightarrow \infty$.

As usual, in order to prove (29), it suffices to show that, for any interval $I$, there exists a constant $\gamma_{I}$ satisfying

$$
\frac{1}{|I|} \int_{I}\left|g_{n}(t)-\gamma_{I}\right| d t \leq c^{\prime}\left(\sum_{k=-n}^{n}\left|b_{k}\right|^{2}\right)^{1 / 2}
$$

For an interval $I$, if $2 m \pi<|I| \leq 2(m+1) \pi$ for some $m \geq 1$, then

$$
\left(\frac{1}{|I|} \int_{I}\left|g_{n}(t)\right| d t\right)^{2} \leq \frac{1}{|I|} \int_{I}\left|g_{n}(t)\right|^{2} d t \leq \frac{m+1}{2 m \pi} \int_{-\pi}^{\pi}\left|g_{n}(t)\right|^{2} d t \leq c \sum_{k=-n}^{n}\left|b_{k}\right|^{2} .
$$

If $\pi / 4 \leq|I| \leq 2 \pi$, we have a similar estimate.

In what follows we assume that $2 \pi /(m+1)<|I| \leq 2 \pi / m$ for some $m \geq 8$. For such an interval, if $m \geq n$, then choosing $t_{I}$ to be one of the end points of $I$, we have

$$
\left|g_{n}(t)-g_{n}\left(t_{I}\right)\right|^{2} \leq \sum_{k=-n}^{n}\left|b_{k}\right|^{2} \sum_{k=-n}^{n}\left|\lambda_{k}\right|^{2}\left|\mathcal{E}_{k}(t)-\mathcal{E}_{k}\left(t_{I}\right)\right|^{2}
$$

and by Lemma 2.6,

$$
\left|g_{n}(t)-g_{n}\left(t_{I}\right)\right|^{2} \leq c \sum_{k=-n}^{n}\left|b_{k}\right|^{2} \sum_{k=-n}^{n}\left|\lambda_{k}\right|^{2}|k|^{2 \delta}\left|t-t_{I}\right|^{2 \delta}
$$


where $\delta=\gamma(\alpha, \beta)-1$ if $\gamma(\alpha, \beta)$ is finite and $1<\gamma(\alpha, \beta) \leq 2$, and $\delta=1$ otherwise. From assumption (5), $\sum_{N \leq|n| \leq 2 N}\left|\lambda_{n}\right|^{2}=O(1)$, which implies $\sum_{k=-n}^{n}\left|\lambda_{k}\right|^{2}|k|^{2 \delta} \leq c n^{2 \delta}$ by Proposition $1.6((\mathrm{ii}) \Rightarrow$ (i) with $b=a=2 \delta>0)$. Therefore

$$
\left|g_{n}(t)-g_{n}\left(t_{I}\right)\right|^{2} \leq c \sum_{|k| \leq n}\left|b_{k}\right|^{2}(n|I|)^{2 \delta} \leq c^{\prime} \sum_{|k| \leq n}\left|b_{k}\right|^{2},
$$

and (30) is true with $\gamma_{I}=g_{n}\left(t_{I}\right)$.

If $m<n$, we again choose $t_{I}$ to be one of the end points of $I$ to get

$$
\left|g_{n}(t)-g_{m}\left(t_{I}\right)\right| \leq\left|g_{m}(t)-g_{m}\left(t_{I}\right)\right|+\left|\sum_{m<|k| \leq n} \lambda_{k} b_{k} \mathcal{E}_{k}(t)\right| .
$$

Hence by what has been verified,

$$
\frac{1}{|I|} \int_{I}\left|g_{n}(t)-g_{m}\left(t_{I}\right)\right| d t \leq c^{\prime}\left(\sum_{k=-m}^{m}\left|b_{k}\right|^{2}\right)^{1 / 2}+F_{m, n},
$$

where $F_{m, n}=|I|^{-1} \int_{I}\left|\sum_{m<|k| \leq n} \lambda_{k} b_{k} \mathcal{E}_{k}(t)\right| d t$. But for $F_{m, n}$, we first note

$$
\begin{aligned}
F_{m, n}^{2} & \leq \frac{1}{|I|} \int_{I}\left|\sum_{m<|k| \leq n} \lambda_{k} b_{k} \mathcal{E}_{k}(t)\right|^{2} d t \\
& \leq \sum_{m<|k| \leq n} \sum_{m<|j| \leq n}\left|\lambda_{k} b_{k} \overline{\lambda_{j} b_{j}}\right| \frac{1}{|I|}\left|\int_{I} \mathcal{E}_{k}(t) \overline{\mathcal{E}_{j}(t)} d t\right| .
\end{aligned}
$$

By symmetry, it suffices to evaluate the part $\sum_{m<|k| \leq n} \sum_{m<|j| \leq|k|}$, and for these $j, k$, $2 \pi|I|^{-1} \leq m+1 \leq|j|$, and by Lemma 2.7 ,

$$
|I|^{-1}\left|\int_{I} \mathcal{E}_{k}(t) \overline{\mathcal{E}_{j}(t)} d t\right| \leq c\left(|j|+|I|^{-1}\right) /|k| \leq 2 c|j| /|k| .
$$

Thus the evaluation of $F_{m, n}^{2}$ is reduced to showing the following inequality:

$$
S_{m, n}:=\sum_{m<|k| \leq n} \sum_{m<j|\leq| k \mid}\left|\lambda_{k} b_{k} \overline{\lambda_{j}} b_{j}\right| \frac{|j|}{|k|} \leq c \sum_{m<|k| \leq n}\left|b_{k}\right|^{2} .
$$

For the purpose, we rewrite $S_{m, n}$ as

$$
\begin{aligned}
S_{m, n} & \leq \frac{1}{2} \sum_{m<|k| \leq n} \sum_{m<|j| \leq|k|}\left(\left|\lambda_{j} b_{k}\right|^{2}+\left|\lambda_{k} b_{j}\right|^{2}\right) \frac{|j|}{|k|} \\
& =\frac{1}{2} \sum_{m<|k| \leq n} \frac{\left|b_{k}\right|^{2}}{|k|} \sum_{m<|j| \leq|k|}\left|\lambda_{j}\right|^{2}|j|+\frac{1}{2} \sum_{m<j \mid \leq n}\left|b_{j}\right|^{2}|j| \sum_{|j| \leq|k| \leq n} \frac{\left|\lambda_{k}\right|^{2}}{|k|} .
\end{aligned}
$$

Since assumption (5) $(q=2)$ implies

$$
\sum_{|j| \leq|k|}\left|\lambda_{j}\right|^{2}|j| \leq c|k| \text { and } \sum_{|k| \geq|j|} \frac{\left|\lambda_{k}\right|^{2}}{|k|} \leq c|j|^{-1},
$$


by Proposition 1.6 ((ii) $\Rightarrow$ (i) and (ii) $\Rightarrow$ (iv) with $b=a=\delta=1$ ). Incorporating these into (32) proves that $S_{m, n} \leq c^{\prime} \sum_{m<|k| \leq n}\left|b_{k}\right|^{2}$; furthermore $F_{m, n} \leq c\left(\sum_{m<|k| \leq n}\left|b_{k}\right|^{2}\right)^{1 / 2}$. Inserting this into (31) proves (30) with $\gamma_{I}=g_{m}\left(t_{I}\right)$.

The proof of Theorem 1.3 is completed.

\subsection{Proof of Theorem 1.4}

We fix a sequence $\left\{b_{n}\right\}_{n=-\infty}^{\infty} \in \ell^{q^{\prime}}, q^{-1}+q^{\prime-1}=1$, and for $n=1,2, \ldots$, define $g_{n}$ as in (27). By Proposition 1.2 and Lemma 1.1,

$$
\left|\sum_{k=-n}^{n} \lambda_{k} b_{k} c_{k}(f)\right|=\left|\sum_{k=-n}^{n} \lambda_{k} b_{k} \mathcal{L}_{\overline{\mathcal{E}}_{k}}(f)\right|=\left|\mathcal{L}_{g_{n}}(f)\right| \leq c\left\|g_{n}\right\|_{\Lambda_{p^{-1}-1}}\|f\|_{H^{p}} .
$$

In order to prove (6), it suffices to show that there is a constant $c^{\prime}$ independent of $n$ and $\left\{b_{k}\right\} \in \ell^{q^{\prime}}$ such that

$$
\left\|g_{n}\right\|_{\Lambda_{p^{-1}-1}} \leq c^{\prime}\left\|\left\{b_{k}\right\}\right\|_{q^{\prime}}
$$

Assume $m-1<\delta:=p^{-1}-1<m \leq \gamma(\alpha, \beta)-1$. From (27) we have, for $h \neq 0$,

$$
\left|g_{n}^{(m-1)}(t+h)-g_{n}^{(m-1)}(t)\right| \leq \sum_{k=-n}^{n}\left|\lambda_{k} b_{k}\right|\left|\mathcal{E}_{k}^{(m-1)}(t+h)-\mathcal{E}_{k}^{(m-1)}(t)\right| .
$$

If $n \leq|h|^{-1}$, we apply Lemma 2.6 for $m \leq \gamma(\alpha, \beta)-1$ to get an upper bound of $\mid g_{n}^{(m-1)}(t+$ $h)-g_{n}^{(m-1)}(t) \mid$ as a multiple of

$$
\sum_{k=-n}^{n}\left|\lambda_{k} b_{k}\right||k|^{m}|h| \leq|h|\left\|\left\{b_{k}\right\}\right\|_{q^{\prime}}\left(\sum_{k=-n}^{n}\left|\lambda_{k}\right|^{q}|k|^{m q}\right)^{1 / q} .
$$

Since $q\left(1-p^{-1}\right)=a-b$, where $a=q\left(m+1-p^{-1}\right)>0, b=m q$, condition (7) and Proposition $1.6(($ ii $) \Rightarrow$ (i)) give

$$
\sum_{k=-n}^{n}\left|\lambda_{k}\right|^{q}|k|^{m q} \leq c n^{q\left(m+1-p^{-1}\right)} \leq c|h|^{q\left(p^{-1}-m-1\right)} \quad \text { for } n \leq|h|^{-1} .
$$

Substituting this into (35) yields

$$
\left|g_{n}^{(m-1)}(t+h)-g_{n}^{(m-1)}(t)\right| \leq c\left\|\left\{b_{k}\right\}\right\|_{q^{\prime}}|h|^{p^{-1}-m} .
$$

If $n>|h|^{-1}$, the summation of those terms in (34) for $|k| \leq|h|^{-1}$ has the same bound $c\left\|\left\{b_{k}\right\}\right\|_{q^{\prime}}|h|^{p^{-1}-m}$ as above and the summation of the terms for $|h|^{-1}<|k| \leq n$, in virtue of Corollary 2.5 , is dominated by

$$
\begin{aligned}
& \sum_{|h|^{-1}<|k| \leq n}\left|\lambda_{k} b_{k}\right|\left(\left|\mathcal{E}_{k}^{(m-1)}(t+h)\right|+\left|\mathcal{E}_{k}^{(m-1)}(t)\right|\right) \\
& \leq \sum_{|h|^{-1<|k| \leq n}}\left|\lambda_{k} b_{k}\right||k|^{m-1} \leq c\left\|\left\{b_{k}\right\}\right\|_{q^{\prime}}\left(\sum_{|h|^{-1}<|k| \leq n}\left|\lambda_{k}\right|^{q}|k|^{q(m-1)}\right)^{1 / q} .
\end{aligned}
$$


Since

$$
q(m-1)=q\left(p^{-1}-1\right)-q\left(p^{-1}-m\right) \quad \text { and } \quad q\left(p^{-1}-m\right)>0,
$$

condition (7) and Proposition $1.6((\mathrm{ii}) \Rightarrow$ (iv)) give

$$
\sum_{|h|^{-1}<|k| \leq n}\left|\lambda_{k}\right|^{q}|k|^{q(m-1)} \leq c\left(|h|^{-1}\right)^{-q\left(p^{-1}-m\right)}=c|h|^{q\left(p^{-1}-m\right)} .
$$

Substituting this into the previous evaluation yields an upper bound of the summation of the terms in (34) for $|h|^{-1}<|k| \leq n$ as $c\left\|\left\{b_{k}\right\}\right\|_{q^{\prime}}|h|^{p^{-1}-m}$. Thus (36) is proved to be true for all $n$ and $h$, so that (33) is shown whenever

$$
m-1<\delta:=p^{-1}-1<m \leq \gamma(\alpha, \beta)-1 .
$$

Next we consider the case when $\gamma(\alpha, \beta)$ is finite and

$$
m-1<\delta:=p^{-1}-1<\gamma(\alpha, \beta)-1<m \text {. }
$$

Similarly to (35), we apply (23) in (34) to obtain

$$
\begin{aligned}
& \left|g_{n}^{(m-1)}(t+h)-g_{n}^{(m-1)}(t)\right| \\
& \quad \leq c|h|^{\gamma(\alpha, \beta)-m}\left\|\left\{b_{k}\right\}\right\|_{q^{\prime}}\left(\sum_{k=-n}^{n}\left|\lambda_{k}\right|^{q}|k|^{q(\gamma(\alpha, \beta)-1)}\right)^{1 / q} .
\end{aligned}
$$

Since $q\left(1-p^{-1}\right)=a-b$, where $a=q\left(\gamma(\alpha, \beta)-p^{-1}\right)>0, b=q(\gamma(\alpha, \beta)-1)$, condition (7) and Proposition $1.6((\mathrm{ii}) \Rightarrow$ (i)) give

$$
\sum_{k=-n}^{n}\left|\lambda_{k}\right|^{q}|k|^{q(\gamma(\alpha, \beta)-1)} \leq c n^{q\left(\gamma(\alpha, \beta)-p^{-1}\right)} \leq c|h|^{q\left(p^{-1}-\gamma(\alpha, \beta)\right)}
$$

for $n \leq|h|^{-1}$, and substituting this into (38) proves (36) again. If $n>|h|^{-1}$, we also break the summation in (34) into two parts according to $|k| \leq|h|^{-1}$ and $|h|^{-1}<|k| \leq n$, where the first part has the same bound $c\left\|\left\{b_{k}\right\}\right\|_{q^{\prime}}|h|^{p^{-1}-m}$ as just proved and the second part is dealt with by the same way as in (37). That means (36) is true for all $n$ and $h$, and hence (33) is proved for $m-1<\delta:=p^{-1}-1<\gamma(\alpha, \beta)-1<m$.

Finally, we prove (33) for

$$
\delta:=p^{-1}-1=m<\gamma(\alpha, \beta)-1 .
$$

It is noted that the verification from (35) to (36) for $n \leq|h|^{-1}$ does not work when $p^{-1}=m+1$. We shall need to evaluate the second order difference of $g_{n}^{(m-1)}$, which is also sufficient by our definition about $\Lambda_{\delta}$ for $\delta=m$. From (27) it follows, for $h \neq 0$, that $\left|g_{n}^{(m-1)}(t+h)-2 g_{n}^{(m-1)}(t)+g_{n}^{(m-1)}(t-h)\right|$ is bounded by

$$
\sum_{k=-n}^{n}\left|\lambda_{k} b_{k}\right|\left|\mathcal{E}_{k}^{(m-1)}(t+h)-2 \mathcal{E}_{k}^{(m-1)}(t)+\mathcal{E}_{k}^{(m-1)}(t-h)\right| .
$$


If $1 \leq m<\gamma(\alpha, \beta)-2$, this is dominated by $c \sum_{k=-n}^{n}\left|\lambda_{k} b_{k}\right|\left|\mathcal{E}_{k}^{(m+1)}(\xi)\right||h|^{2}$; furthermore, by virtue of Corollary 2.5, by

$$
c^{\prime}|h|^{2} \sum_{k=-n}^{n}\left|\lambda_{k} b_{k}\right||k|^{m+1} \leq c^{\prime}|h|^{2}\left\|\left\{b_{k}\right\}\right\|_{q^{\prime}}\left(\sum_{k=-n}^{n}\left|\lambda_{k}\right|^{q}|k|^{q(m+1)}\right)^{1 / q} .
$$

Since $q\left(1-p^{-1}\right)=-q m=a-b$, where $a=q>0, b=q(m+1)$, condition (7) and Proposition $1.6((\mathrm{ii}) \Rightarrow(\mathrm{i}))$ give

$$
\sum_{k=-n}^{n}\left|\lambda_{k}\right|^{q}|k|^{q(m+1)} \leq c n^{q} \leq c|h|^{-q} \text { for } n \leq|h|^{-1} .
$$

Substituting this into (40) yields, for $n \leq|h|^{-1}$,

$$
\left|g_{n}^{(m-1)}(t+h)-2 g_{n}^{(m-1)}(t)+g_{n}^{(m-1)}(t-h)\right| \leq c\left\|\left\{b_{k}\right\}\right\|_{q^{\prime}}|h| .
$$

If $\gamma(\alpha, \beta)$ is finite and $\gamma(\alpha, \beta)-2 \leq m<\gamma(\alpha, \beta)-1$, we note that

$$
\left|\mathcal{E}_{k}^{(m-1)}(t+h)-2 \mathcal{E}_{k}^{(m-1)}(t)+\mathcal{E}_{k}^{(m-1)}(t-h)\right|=\left|\mathcal{E}_{k}^{(m)}\left(\xi_{1}\right)-\mathcal{E}_{k}^{(m)}\left(\xi_{2}\right)\right||h|
$$

by the mean-value theorem, where $\xi_{1}$ and $\xi_{2}$ lay between $t-h$ and $t+h$; furthermore, by (23) this is bounded by

$$
c n^{\gamma(\alpha, \beta)-1}|h|^{\gamma(\alpha, \beta)-m-1}|h|=c n^{\gamma(\alpha, \beta)-1}|h|^{\gamma(\alpha, \beta)-m} .
$$

Hence the expression in (39) is dominated by a multiple of

$$
\sum_{k=-n}^{n}\left|\lambda_{k} b_{k}\right||k|^{\gamma(\alpha, \beta)-1}|h|^{\gamma(\alpha, \beta)-m}
$$

which has the same bound as in (38), and also the bound $c\left\|\left\{b_{k}\right\}\right\|_{q^{\prime}}|h|^{p^{-1}-m}=c\left\|\left\{b_{k}\right\}\right\|_{q^{\prime}}|h|$ for $n \leq|h|^{-1}$ as in (36). Thus (41) is shown to be true for $n \leq|h|^{-1}$.

If $n>|h|^{-1}$, the summation of the terms for $|k| \leq|h|^{-1}$ in (39) has the same bound as in (41), and the summation of those for $|h|^{-1}<|k| \leq n$ is dealt with by the same way as in (37) to obtain its bound $c\left\|\left\{b_{k}\right\}\right\|_{q^{\prime}}|h|^{p^{-1}-m}=c\left\|\left\{b_{k}\right\}\right\|_{q^{\prime}}|h|$. Therefore (41) is verified for all $n$ and $h$, and hence (33) is proved for $\delta:=p^{-1}-1=m<\gamma(\alpha, \beta)-1$.

The proof of Theorem 1.4 is completed.

Acknowledgements

Not applicable.

Funding

The research is supported by the National Natural Science Foundation of China (Nos. 11601352, 11571261, 11371258)

Availability of data and materials

Not applicable.

Competing interests

None of the authors have any competing interests in the manuscript. 
Authors' contributions

All authors contributed equally and significantly in conducting this research work and writing this paper. All authors read and approved the final manuscript.

\section{Author details}

${ }^{1}$ Department of Mathematics, Capital Normal University, Beijing, China. ${ }^{2}$ Elementary Education College, Capital Normal University, Beijing, China. ${ }^{3}$ Department of Mathematics, Shanghai Normal University, Shanghai, China.

\section{Publisher's Note}

Springer Nature remains neutral with regard to jurisdictional claims in published maps and institutional affiliations.

Received: 3 March 2019 Accepted: 21 January 2020 Published online: 11 February 2020

\section{References}

1. Duren, P.L.: Theory of $H^{P}$ Spaces. Academic Press, New York (1970)

2. Duren, P.L., Shields, A.L.: Coefficient multipliers of $H^{P}$ and $B^{P}$ spaces. Pac. J. Math. 32, 69-78 (1970)

3. Erdélyi, A., Magnus, W., Oberhettinger, F., Tricomi, F.G.: Higher Transcendental Functions, vols. I-III. McGraw-Hill, New York (1953)

4. Hardy, G.H., Littlewood, J.E.: Notes on the theory of series (XX): generalizations of a theorem of Paley. Q. J. Math. 8, 161-171 (1937)

5. Hardy, G.H., Littlewood, J.E.: Theorems concerning mean values of analytic or harmonic functions. Q. J. Math. 12 221-256 (1941)

6. Kanjin, Y.: Hardy's inequalities for Hermite and Laguerre expansions revisited. J. Math. Soc. Jpn. 63, 753-767 (2011)

7. Kanjin, Y., Sato, K.: Paley's inequality for the Jacobi expansions. Bull. Lond. Math. Soc. 33, 483-491 (2001)

8. Li, Z.-K.: Conjugate Jacobi series and conjugate functions. J. Approx. Theory 86, 179-196 (1996)

9. Li, Z.-K., Liu, L.-M.: Uncertainty principles for Jacobi expansions. J. Math. Anal. Appl. 286, 652-663 (2003)

10. Li, Z.-K., Shi, Y.-H.: Multipliers of Hardy spaces associated with generalized Hermite expansions. Constr. Approx. 39 517-540 (2014)

11. Li, Z.-K., Yu, Y.-F., Shi, Y.-H.: The Hardy inequality for Hermite expansions. J. Fourier Anal. Appl. 21, $267-280$ (2015)

12. Sledd, W.T.: On multipliers of $H^{P}$ spaces. Indiana Univ. Math. J. 27, 797-803 (1978)

13. Stein, E.M., Zygmund, A.: Boundedness of translation invariant operators on Hölder spaces and $L^{p}$-spaces. Ann. Math. 85, 337-349 (1967)

14. Szegö, G.: Orthogonal Polynomials. Am. Math. Soc. Colloq. Publ., vol. 23. Am. Math. Soc., Providence (1975)

\section{Submit your manuscript to a SpringerOpen ${ }^{\circ}$ journal and benefit from:}

- Convenient online submission

- Rigorous peer review

Open access: articles freely available online

- High visibility within the field

- Retaining the copyright to your article

Submit your next manuscript at $\boldsymbol{~ s p r i n g e r o p e n . c o m ~}$ 